JOURNAL OF

FUNCTION SPACES AND APPLICATIONS

Volume 5, Number 1 (2007), 89-101 (c) 2007, Scientific Horizon

http://www.jfsa.net

\title{
On variational formulations for functional differential equations
}

\section{A. Kolesnikova, A. M. Popov and V. M. Savchin}

(Communicated by Vladimir Stepanov)

2000 Mathematics Subject Classification. 34Jxx, 34Kxx, 34A30.

Keywords and phrases. Functional differential equations, inverse problem of the calculus of variations, conditions of potentiality, variational principles.

Abstract. Necessary and sufficient conditions for the existence of integral variational principles for boundary value problems for given ordinary and partial functional differential equations are obtained. Examples are given illustrating the results.

\section{Introduction}

By the problem of construction of integral variational principles for a system of equations of some given model we mean the construction of functionals for which the set of critical (extremal or stationary) points coincides with the set of the solutions of the given system.

The search of a functional $F$, that admits the given equations as its Euler-Lagrange equations is known as the classical inverse problem of the calculus of variations. Since the end of the XIXth century there has been a 
great deal of activity in this field (see Helmholtz [4], Volterra [13], Santilli [9], Tonti [12], Filippov, Savchin and Shorokhov [2] and refs. therein).

In the course of a long time advantages of the variational principles have been mainly used for ordinary and partial differential equations with the so-called potential operators [2].

There is a practical need to develop different approaches to the construction of integral variational principles for equations with deviating arguments.

The first known Euler-Lagrange equations with deviating arguments corresponding to some variational problems have been considered by El'sgol'tz [1], Hughes [5], Sabbagh [8] and Kamenskii [6].

The main aim of this paper is to find out necessary and sufficient conditions under which the given ordinary and partial functional differential equations with appropriate boundary conditions admit variational formulation.

The reader should keep in mind that most of the results can be formulated under weaker smoothness conditions.

\section{Certain auxiliary notations and definitions}

Let $U, V$ be normed linear spaces over the field of real numbers $\mathbb{R}$, and $O_{U}, O_{V}$ be their zero elements.

Take any operator $N: D(N) \rightarrow R(N)$, where $D(N) \subseteq U, R(N) \subseteq V$. A limit

$$
\lim _{\epsilon \rightarrow 0} \frac{1}{\epsilon}[N(u+\epsilon h)-N(u)]=\delta N(u, h), u \in D(N),(u+\epsilon h) \in D(N),
$$

if it exists, is called the Gâteaux differential of $N$ at the point $u$. If it is linear relative to $h$, then the operator $\delta N(u, \cdot): U \rightarrow V$ is called the Gâteaux derivative of $N$ at $u$ and will be denoted by $N_{u}^{\prime}$. Its domain of definition $D\left(N_{u}^{\prime}\right)$ consists of the elements $h \in U$ such that $(u+\epsilon h) \in D(N)$ for all $\epsilon$ sufficiently small.

Let us consider the equation

$$
N(u)=O_{V}, \quad u \in D(N)
$$

with the Gâteaux differentiable operator $N$, and a convex set $D(N)$.

In order to consider the existence of its variational formulation we need a non-degenerate bilinear form

$$
\langle\cdot, \cdot\rangle: V \times U \rightarrow R
$$


Definition. The operator $N: D(N) \rightarrow V$ is said to be potential on the set $D(N)$ relative to a given bilinear form $\langle\cdot, \cdot\rangle: V \times U \rightarrow R$, if there exists a functional $F_{N}: D\left(F_{N}\right)=D(N) \rightarrow R$ such that

$$
\delta F_{N}[u, h]=\langle N(u), h\rangle \quad \forall u \in D(N), \quad \forall h \in D\left(N_{u}^{\prime}\right) .
$$

The functional $F_{N}$ is called the potential of the operator $N$, and in turn the operator $N$ is called the gradient of the functional $F_{N}$. As it is known Volterra [13] the condition for potentiality of the operator $N$ takes the form

$$
\left\langle N_{u}^{\prime} h, g\right\rangle=\left\langle N_{u}^{\prime} g, h\right\rangle \quad \forall u \in D(N), \quad \forall h, g \in D\left(N_{u}^{\prime}\right) .
$$

Under this condition the potential $F_{N}$ is given by

$$
F_{N}[u]=\int_{0}^{1}\left\langle N\left(u_{0}+\lambda\left(u-u_{0}\right)\right), u-u_{0}\right\rangle d \lambda+\text { const },
$$

where $u_{0}$ is a fixed element of $\mathrm{D}(\mathrm{N})$.

\section{The potentiality conditions for ordinary functional differential equations}

Let us consider the equation

$$
\begin{gathered}
N(u) \equiv F\left(t, u\left(\omega_{i}\left(\gamma_{k}(t)\right)\right), u^{\prime}\left(\omega_{i}\left(\gamma_{k}(t)\right)\right), u^{\prime \prime}\left(\omega_{i}\left(\gamma_{k}(t)\right)\right)\right)=0 \\
i, k=\overline{0, n} ; \quad t \in\left(t_{1}, t_{2}\right),
\end{gathered}
$$

where $\omega_{0}(t) \equiv t, \gamma_{0}(t) \equiv t ; \omega_{i}(t)$ and $\gamma_{i}(t)$ are mutually inverse functions; $\omega_{i}(t), \gamma_{i}(t) \in C^{3}\left[t_{1}, t_{2}\right], \omega(t) \leq t, \omega^{\prime}(t)>0 \forall t \in\left[t_{1}, t_{2}\right] ; F \in C^{3}\left(R^{m}\right), m=$ $3\left(n^{2}+n\right)+4$.

Let us denote $\alpha_{j}=\min _{i, k} \omega_{i}\left(\gamma_{k}\left(t_{j}\right)\right), \beta_{j}=\max _{i, k} \omega_{i}\left(\gamma_{k}\left(t_{j}\right)\right), j=1,2$. We assume that $\beta_{1}<\alpha_{2}$. The functions $\varphi_{i}(i=1,2)$, belonging to the class of functions $C^{2}$, are given on the sets $E_{j}=\left[\alpha_{j}, \beta_{j}\right], j=1,2$.

Let us denote by $U$ the set of all absolutely continuous functions on $\left[\alpha_{1}, \beta_{2}\right]$ for which the first and the second derivatives exist and are almost everywhere bounded on $\left[\alpha_{1}, \beta_{2}\right]$.

A function $u(t) \in U$, satisfying the equation (4) almost everywhere on $\left[\alpha_{1}, \beta_{2}\right]$, is called its solution.

The domain of definition of the given operator $N$ is defined by the equality

$$
D(N)=\left\{u \in U: u(t)=\varphi_{j}(t), t \in E_{j}, j=1,2\right\},
$$


where $\varphi_{j}(t)$ are certain given functions, $\varphi_{j}(t) \in C^{2}$.

We define the bilinear form by the relation

$$
\langle v, g\rangle=\int_{t_{1}}^{t_{2}} v(t) g(t) d t
$$

Theorem 1. In order that the operator $N$ (4) be potential on $D(N)$ (5) relative to the bilinear form (6), it is necessary and sufficient that $\forall t \in\left(t_{1}, t_{2}\right)$

$$
\begin{aligned}
& \text { (7) } \quad\left[\frac{\partial F}{\partial u_{i k}}\right]_{k i} \frac{d \omega_{k}\left(\gamma_{i}(t)\right)}{d t}-\frac{d}{d t}\left(\left[\frac{\partial F}{\partial u_{i k}^{\prime}}\right]_{k i} \frac{d \omega_{k}\left(\gamma_{i}(t)\right)}{d t}\right) \\
& +\frac{d^{2}}{d t^{2}}\left(\left[\frac{\partial F}{\partial u_{i k}^{\prime \prime}}\right]_{k i} \frac{d \omega_{k}\left(\gamma_{i}(t)\right)}{d t}\right)=\frac{\partial F}{\partial u_{k i}}, \\
& (8) \quad-\left[\frac{\partial F}{\partial u_{i k}^{\prime}}\right]_{k i}\left(\frac{d \omega_{k}\left(\gamma_{i}(t)\right)}{d t}\right)^{2}+\frac{d}{d t}\left(\left[\frac{\partial F}{\partial u_{i k}^{\prime \prime}}\right]_{k i} \frac{d \omega_{k}\left(\gamma_{i}(t)\right)}{d t}\right) \frac{d \omega_{k}\left(\gamma_{i}(t)\right)}{d t} \\
& +\frac{d}{d t}\left(\left[\frac{\partial F}{\partial u_{i k}^{\prime \prime}}\right]_{k i}\left(\frac{d \omega_{k}\left(\gamma_{i}(t)\right)}{d t}\right)^{2}\right)=\frac{\partial F}{\partial u_{k i}^{\prime}}, \\
& \text { (9) } \quad\left[\frac{\partial F}{\partial u_{i k}^{\prime \prime}}\right]_{k i}\left(\frac{d \omega_{k}\left(\gamma_{i}(t)\right)}{d t}\right)^{3}=\frac{\partial F}{\partial u_{k i}^{\prime \prime}},
\end{aligned}
$$

where $u_{i k}=u\left(\omega_{i}\left(\gamma_{k}(t)\right),[\Psi]_{i k} \equiv \Psi\left(s, u\left(\omega_{p}\left(\gamma_{q}(s)\right)\right) \ldots\right), s=\omega_{i}\left(\gamma_{k}(t)\right)\right.$, $p, q=\overline{0, n}, i, k=\overline{0, n}$.

Proof. We shall verify that under the conditions (7)-(9) the Volterra's criterion of potentiality (2), where

$$
D\left(N_{u}^{\prime}\right)=\left\{h \in U: h=0, t \in E_{1} \cup E_{2}\right\},
$$

will be fulfilled.

Using the Gâteaux derivative of the operator (4), we get

$$
\begin{aligned}
\left\langle N_{u}^{\prime} h, g\right\rangle=\int_{t_{1}}^{t_{2}}\left\{\sum_{i, k=0}^{n}\right. & {\left[\frac{\partial F}{\partial u\left(\omega_{i}\left(\gamma_{k}(t)\right)\right)} h\left(\omega_{i}\left(\gamma_{k}(t)\right)\right)\right.} \\
& +\frac{\partial F}{\partial u^{\prime}\left(\omega_{i}\left(\gamma_{k}(t)\right)\right)} h^{\prime}\left(\omega_{i}\left(\gamma_{k}(t)\right)\right) \\
& \left.\left.+\frac{\partial F}{\partial u^{\prime \prime}\left(\omega_{i}\left(\gamma_{k}(t)\right)\right)} h^{\prime \prime}\left(\omega_{i}\left(\gamma_{k}(t)\right)\right)\right]\right\} g(t) d t
\end{aligned}
$$


Let us perform the following transformations in the right-hand side of the equality (11):

(a) in the first group of terms we use the change of variable of the form $s=\omega_{i}\left(\gamma_{k}(t)\right)$

(b) in the second group of terms we use the change of the variable of integration, then the formula of integration by parts;

(c) in the third group of terms we use the change of the variable of integration, then twice the formula of integration by parts.

Bearing in mind the equality (10) for $h$ and $g$, and going back to the initial notation of the variable of integration, we get

$$
\begin{aligned}
\int_{t_{1}}^{t_{2}} & \frac{\partial F}{\partial u\left(\omega_{i}\left(\gamma_{k}(t)\right)\right)} h\left(\omega_{i}\left(\gamma_{k}(t)\right)\right) g(t) d t \\
= & \int_{t_{1}}^{t_{2}}\left[\frac{\partial F}{\partial u\left(\omega_{i}\left(\gamma_{k}(t)\right)\right)}\right]_{k i} \frac{d \omega_{k}\left(\gamma_{i}(t)\right)}{d t} g\left(\omega_{k}\left(\gamma_{i}(t)\right)\right) h(t) d t \\
\int_{t_{1}}^{t_{2}} & \frac{\partial F}{\partial u^{\prime}\left(\omega_{i}\left(\gamma_{k}(t)\right)\right)} h^{\prime}\left(\omega_{i}\left(\gamma_{k}(t)\right)\right) g(t) d t \\
= & -\int_{t_{1}}^{t_{2}}\left(\frac{d}{d t}\left(\left[\frac{\partial F}{\partial u^{\prime}\left(\omega_{i}\left(\gamma_{k}(t)\right)\right)}\right]_{k i} \frac{d \omega_{k}\left(\gamma_{i}(t)\right)}{d t}\right) g\left(\omega_{k}\left(\gamma_{i}(t)\right)\right)\right. \\
& \left.+\left[\frac{\partial F}{\partial u^{\prime}\left(\omega_{i}\left(\gamma_{k}(t)\right)\right)}\right]_{k i}\left(\frac{d \omega_{k}\left(\gamma_{i}(t)\right)}{d t}\right)^{2} g^{\prime}\left(\omega_{k}\left(\gamma_{i}(t)\right)\right)\right) h(t) d t \\
\int_{t_{1}}^{t_{2}} & \frac{\partial F}{\partial u^{\prime \prime}\left(\omega_{i}\left(\gamma_{k}(t)\right)\right)} h^{\prime \prime}\left(\omega_{i}\left(\gamma_{k}(t)\right)\right) g(t) d t \\
= & \int_{t_{1}}^{t_{2}}\left(\frac{d^{2}}{d t^{2}}\left(\left[\frac{\partial F}{\partial u^{\prime \prime}\left(\omega_{i}\left(\gamma_{k}(t)\right)\right)}\right]_{k i} \frac{d \omega_{k}\left(\gamma_{i}(t)\right)}{d t}\right) g\left(\omega_{k}\left(\gamma_{i}(t)\right)\right)\right. \\
& +\frac{d}{d t}\left(\left[\frac{\partial F}{\partial u^{\prime \prime}\left(\omega_{i}\left(\gamma_{k}(t)\right)\right)}\right]_{k i} \frac{d \omega_{k}\left(\gamma_{i}(t)\right)}{d t}\right)^{d \omega_{k}\left(\gamma_{i}(t)\right)} \frac{\partial{ }^{\prime}\left(\omega_{k}\left(\gamma_{i}(t)\right)\right)}{d t} g^{\prime} \\
& +\frac{d}{d t}\left(\left[\frac{d \omega_{k}\left(\gamma_{i}(t)\right)}{d t}\right)^{2}\right) g^{\prime}\left(\omega_{k}\left(\gamma_{i}(t)\right)\right) \\
& \left.+\left[\frac{\partial F}{\partial u^{\prime \prime}\left(\omega_{i}\left(\gamma_{k}(t)\right)\right)}\right]_{k i}\left(\frac{d \omega_{k}\left(\gamma_{i}(t)\right)}{d t}\right)^{3} g^{\prime \prime}\left(\omega_{k}\left(\gamma_{i}(t)\right)\right)\right) h(t) d t .
\end{aligned}
$$

Now we get

$$
\begin{aligned}
(13)\left\langle N_{u}^{\prime} g, h\right\rangle & \\
=\int_{t_{1}}^{t_{2}}\{ & \sum_{i, k=0}^{n}\left[\frac{\partial F}{\partial u\left(\omega_{i}\left(\gamma_{k}(t)\right)\right)} g\left(\omega_{i}\left(\gamma_{k}(t)\right)\right)+\frac{\partial F}{\partial u^{\prime}\left(\omega_{i}\left(\gamma_{k}(t)\right)\right)} g^{\prime}\left(\omega_{i}\left(\gamma_{k}(t)\right)\right)\right. \\
& \left.\left.+\frac{\partial F}{\partial u^{\prime \prime}\left(\omega_{i}\left(\gamma_{k}(t)\right)\right)} g^{\prime \prime}\left(\omega_{i}\left(\gamma_{k}(t)\right)\right)\right]\right\} h(t) d t .
\end{aligned}
$$


Comparing the expressions (11) and (13), and taking into account the above transformations, we conclude that for the fulfillment of the potentiality criterion it is necessary and sufficient that the relations (7)(9) hold.

The proof of the theorem is completed.

Example 1. Let us consider the equation

$$
\begin{aligned}
(14) N(u) \equiv & a_{1} u(t)+a_{2} u\left(c_{1} t\right)+a_{3} u\left(c_{2} t\right)+\frac{a_{2}}{c_{1}} u\left(\frac{t}{c_{1}}\right)+\frac{a_{3}}{c_{2}} u\left(\frac{t}{c_{2}}\right) \\
& +a_{4} u^{\prime}\left(c_{1} t\right)-\frac{a_{4}}{c_{1}^{2}} u^{\prime}\left(\frac{t}{c_{1}}\right)+a_{5} u^{\prime}\left(\frac{c_{1}}{c_{2}} t\right)-a_{5} \frac{c_{2}^{2}}{c_{1}^{2}} u^{\prime}\left(\frac{c_{2}}{c_{1}} t\right) \\
& +a_{6} u^{\prime \prime}\left(c_{1} t\right)+\frac{a_{6}}{c_{1}^{3}} u^{\prime \prime}\left(\frac{t}{c_{1}}\right)+a_{7} u^{\prime \prime}\left(\frac{c_{1}}{c_{2}} t\right)+a_{7} \frac{c_{2}^{3}}{c_{1}^{3}} u^{\prime \prime}\left(\frac{c_{2}}{c_{1}} t\right)=0, \\
& 0<t_{1}<t<t_{2},
\end{aligned}
$$

where $a_{i}(i=\overline{1,7}), c_{1}$ and $c_{2}$ are constants, such that $0<c_{1}<c_{2}<1$.

In accordance with the accepted notations (4) here we have $\omega_{1}(t)=$ $c_{1} t, \quad \omega_{2}(t)=c_{2} t, \quad \gamma_{1}(t)=\frac{t}{c_{1}}, \quad \gamma_{2}=\frac{t}{c_{2}}, \quad \omega_{1}\left(\gamma_{2}(t)\right)=\frac{c_{1}}{c_{2}} t, \quad \omega_{2}\left(\gamma_{1}(t)\right)=$ $\frac{c_{2}}{c_{1}} t$.

Let us note that if $a_{6} \not \equiv 0$ then the equation (14) is of neutral type, and if $a_{6}=0$, then it is of advanced type (see Kamenskii [6]). It is easy to check that the given operator (14) satisfies the conditions of potentiality (7)-(9). Applying the formula (3) for the potential $F_{N}$ of the operator $N$ we get

$$
\begin{aligned}
F_{N}[u]= & \frac{1}{2} \int_{t_{1}}^{t_{2}}\left[a_{1} u^{2}(t)+a_{2} u\left(c_{1} t\right) u(t)+a_{3} u\left(c_{2} t\right) u(t)\right. \\
& +\frac{a_{2}}{c_{1}} u\left(\frac{t}{c_{1}}\right) u(t)+\frac{a_{3}}{c_{2}} u\left(\frac{t}{c_{2}}\right) u(t)+a_{4} u^{\prime}\left(c_{1} t\right) u(t) \\
& -\frac{a_{4}}{c_{1}^{2}} u^{\prime}\left(\frac{t}{c_{1}}\right) u(t)+a_{5} u^{\prime}\left(\frac{c_{1}}{c_{2}} t\right) u(t)-a_{5} \frac{c_{2}^{2}}{c_{1}^{2}} u^{\prime}\left(\frac{c_{2}}{c_{1}} t\right) u(t) \\
& +a_{6} u^{\prime \prime}\left(c_{1} t\right) u(t)+\frac{a_{6}}{c_{1}^{3}} u^{\prime \prime}\left(\frac{t}{c_{1}}\right) u(t)+a_{7} u^{\prime \prime}\left(\frac{c_{1}}{c_{2}} t\right) u(t) \\
& \left.+a_{7} \frac{c_{2}^{3}}{c_{1}^{3}} u^{\prime \prime}\left(\frac{c_{2}}{c_{1}} t\right) u(t)\right] d t .
\end{aligned}
$$

Integrating by parts, we diminish the second order of derivatives in the integrand, and finally we come to the following potential

$$
\begin{aligned}
F_{N}[u]=\int_{t_{1}}^{t_{2}} & {\left[\frac{a_{1}}{2} u^{2}(t)+a_{2} u(t) u\left(c_{1} t\right)+a_{3} u(t) u\left(c_{2} t\right)+a_{4} u(t) u^{\prime}\left(c_{1} t\right)\right.} \\
& \left.+a_{5} c_{2} u^{\prime}\left(c_{1} t\right) u\left(c_{2} t\right)-\frac{a_{6}}{c_{1}} u^{\prime}(t) u^{\prime}\left(\frac{t}{c_{1}}\right)-a_{7} \frac{c_{2}^{2}}{c_{1}} u^{\prime}\left(c_{1} t\right) u^{\prime}\left(c_{2} t\right)\right] d t \\
& + \text { const. }
\end{aligned}
$$




\section{On variational formulation for partial differential difference equations}

Let us consider the following equation

$$
\begin{aligned}
& N(u) \equiv f\left(x, u_{\alpha}^{(k)}(x, t+\lambda \tau)\right)=0, \quad x=\left(x_{1}, \ldots, x_{m}\right) \in \Omega \\
& t \in\left(t_{0}, t_{1}\right), \quad k=\overline{0, l} ; \quad \lambda=-1,0,1 ; \quad \tau>0 \\
& \alpha=\left(\alpha_{1}, \ldots, \alpha_{m}\right), \quad|\alpha|=\sum_{i=1}^{m} \alpha_{i}, \quad|\alpha|=\overline{0, s}, \quad t_{1}-t_{0}>2 \tau,
\end{aligned}
$$

where $u$ is an unknown function, $f$ is a given smooth function,

$$
u_{\alpha}^{(k)}=\frac{\partial^{k} \partial_{\alpha} u}{\partial t^{k}} ; \quad \partial_{\alpha}=\frac{\partial^{|\alpha|}}{\left(\partial x_{1}\right)^{\alpha_{1}} \ldots\left(\partial x_{m}\right)^{\alpha_{m}}} .
$$

The domain of definition $D(N)$ is given by the equality

$$
\begin{aligned}
D(N)= & \left\{u \in U=C_{x, t}^{s, l}\left(\bar{\Omega} \times\left[t_{0}-\tau, t_{1}+\tau\right]\right):\right. \\
& \frac{\partial^{k} u}{\partial t^{k}}=\varphi_{1 k}(x, t),(x, t) \in E_{1}=\bar{\Omega} \times\left[t_{0}-\tau, t_{0}\right] ; k=\overline{0, l_{0}} \\
& \frac{\partial^{k} u}{\partial t^{k}}=\varphi_{2 k}(x, t),(x, t) \in E_{2}=\bar{\Omega} \times\left[t_{1}, t_{1}+\tau\right] ; k=\overline{0, l_{0}}, \\
& \left.\left.\frac{\partial^{\nu} u}{\partial n_{x}^{\nu}}\right|_{\partial \Gamma_{\tau}}=\psi_{\nu}(x, t), \quad \nu=\overline{0, s_{0}}\right\} .
\end{aligned}
$$

Here $\varphi_{10}, \varphi_{20}, \psi_{\nu}$ are given sufficiently smooth functions, $\varphi_{j k}=$ $\frac{\partial^{k} \varphi_{j 0}}{\partial t^{k}}\left(j=1,2 ; k=\overline{1, l_{o}}\right), \Gamma_{\tau}=\partial \Omega \times\left[t_{0}-\tau, t_{1}+\tau\right]$. The numbers $l_{0}$ and $s_{0}$ depend on $l$ and $s$, respectively. If $l, s$ are even, then $l_{0}=\frac{l}{2}-1, \quad s_{0}=$ $\frac{s}{2}-1$. For odd $l, s$ we set $l_{0}=\frac{l+1}{2}-1, \quad s_{0}=\frac{s+1}{2}-1$.

We shall use the notations

$$
\left(\begin{array}{l}
\alpha \\
\beta
\end{array}\right)=\left\{\begin{array}{l}
\left(\begin{array}{c}
\alpha_{1} \\
\beta_{1}
\end{array}\right) \ldots\left(\begin{array}{c}
\alpha_{m} \\
\beta_{m}
\end{array}\right), \text { if } \alpha_{\mathrm{i}} \geq \beta_{\mathrm{i}} \\
0, \text { if } \alpha_{\mathrm{i}}<\beta_{\mathrm{i}},
\end{array}\right.
$$

and

$$
C_{k}^{\nu}=\left(\begin{array}{l}
k \\
\nu
\end{array}\right)=\frac{k !}{\nu !(k-\nu) !}
$$

for binomial coefficients. 
Assume that there is given the bilinear form

$$
\langle v, g\rangle=\int_{\Omega} \int_{t_{0}}^{t_{1}} v(x, t) g(x, t) d t d x .
$$

Theorem 2. For the potentiality of the operator (15) on the given set (16) with respect to the bilinear form (17), it is necessary and sufficient that the following conditions hold

$$
\begin{aligned}
& \left.\sum_{k=0}^{l} \sum_{|\alpha|=0}^{s}(-1)^{|\alpha|+k} C_{k}^{\nu}\left(\begin{array}{c}
\alpha \\
\beta
\end{array}\right) D_{t}^{k-\nu} D_{\alpha-\beta}\left(\frac{\partial f}{\partial u_{\alpha}^{(k)}(x, t-\lambda \tau)}\right)\right|_{t \rightarrow t+\lambda \tau} \\
& \quad=\frac{\partial f}{\partial u_{\beta}^{(\nu)}(x, t-\lambda \tau)}
\end{aligned}
$$

$\forall u \in D(N), \forall x \in \Omega, \forall t \in\left(t_{0}, t_{1}\right), \lambda=-1,0,1, \nu=\overline{0, l},|\beta|=\overline{0, s}$ where the notation $\left.(\ldots)\right|_{t \rightarrow t+\lambda \tau}$ means that in the expression within the parentheses one has to change $t$ by $t+\lambda \tau$.

Proof. Taking into account formulas (15),(17), we get

$$
\begin{aligned}
& \left\langle N_{u}^{\prime} h, g\right\rangle \\
& =\int_{t_{0}}^{t_{1}} \int_{\Omega} \sum_{\lambda=-1}^{1} \sum_{k=0}^{l} \sum_{|\alpha|=0}^{s} \frac{\partial f}{\partial u_{\alpha}^{(k)}(x, t-\lambda \tau)} h_{\alpha}^{(k)}(x, t-\lambda \tau) g(x, t) d x d t .
\end{aligned}
$$

By integrating by parts and taking into account that the functions $g, h \in D\left(N_{u}^{\prime}\right)$ are subject to the conditions

$$
\begin{aligned}
\frac{\partial^{k} g}{\partial t^{k}} & =\frac{\partial^{k} h}{\partial t^{k}}=0, \quad(x, t) \in E_{1} \cup E_{2}, k=\overline{0, l_{0}}, \\
\left.\frac{\partial^{\nu} g}{\partial n_{x}^{\nu}}\right|_{\Gamma_{\tau}} & =\left.\frac{\partial^{\nu} h}{\partial n_{x}^{\nu}}\right|_{\Gamma_{\tau}}=0, \quad \nu=\overline{0, s_{0}},
\end{aligned}
$$

we obtain from (19)

$$
\begin{aligned}
\left\langle N_{u}^{\prime} h, g\right\rangle= & \int_{t_{0}}^{t_{1}} \int_{\Omega} \sum_{\lambda=-1}^{1} \sum_{k=0}^{l} \sum_{|\alpha|=0}^{s}(-1)^{|\alpha|+k} D_{t}^{k} D_{\alpha} \\
& \times\left(\frac{\partial f}{\partial u_{\alpha}^{(k)}(x, t-\lambda \tau)} g(x, t)\right) h(x, t-\lambda \tau) d x d t
\end{aligned}
$$


where $D_{t}^{k}$ is the total derivative of order $k$ with respect to the variable $t ; D_{\alpha}$ is the total derivative corresponding to the multiindex $\alpha$.

From here by using the Leibnitz formula there follows that

$$
\begin{aligned}
\left\langle N_{u}^{\prime} h, g\right\rangle= & \int_{t_{0}}^{t_{1}} \int_{\Omega} \sum_{\lambda=-1}^{1} \sum_{k, \nu=0}^{l} \sum_{|\alpha|,|\beta|=0}^{s}(-1)^{|\alpha|+k} C_{k}^{\nu}\left(\begin{array}{l}
\alpha \\
\beta
\end{array}\right) \\
& \times D_{t}^{k-\nu} D_{\alpha-\beta}\left(\frac{\partial f}{\partial u_{\alpha}^{(k)}(x, t-\lambda \tau)}\right) \\
& \times g_{\beta}^{(\nu)}(x, t) h(x, t-\lambda \tau) d x d t .
\end{aligned}
$$

By using the change $t-\lambda \tau=t^{\prime}$, from (20) we get

$$
\begin{aligned}
\left\langle N_{u}^{\prime} h, g\right\rangle= & \sum_{\lambda=-1}^{1} \int_{t_{0}-\lambda \tau}^{t_{1}-\lambda \tau} \int_{\Omega} \sum_{k, \nu=0}^{l} \sum_{|\alpha|,|\beta|=0}^{s}(-1)^{|\alpha|+k} C_{k}^{\nu}\left(\begin{array}{l}
\alpha \\
\beta
\end{array}\right) \\
& \times\left. D_{t^{\prime}}^{k-\nu} D_{\alpha-\beta}\left(\frac{\partial f}{\partial u_{\alpha}^{(k)}(x, t-\lambda \tau)}\right)\right|_{t \rightarrow t^{\prime}+\lambda \tau} \\
& \times g_{\beta}^{(\nu)}\left(x, t^{\prime}+\lambda \tau\right) h\left(x, t^{\prime}\right) d x d t^{\prime} .
\end{aligned}
$$

Denoting $t^{\prime}$ by $t$, we obtain from (21)

$$
\begin{aligned}
\left\langle N_{u}^{\prime} h, g\right\rangle= & \sum_{\lambda=-1}^{1} \int_{t_{0}-\lambda \tau}^{t_{1}-\lambda \tau} \int_{\Omega} \sum_{k, \nu=0}^{l} \sum_{|\alpha|,|\beta|=0}^{s}(-1)^{|\alpha|+k} C_{k}^{\nu}\left(\begin{array}{c}
\alpha \\
\beta
\end{array}\right) \\
& \times\left. D_{t^{\prime}}^{k-\nu} D_{\alpha-\beta}\left(\frac{\partial f}{\partial u_{\alpha}^{(k)}(x, t-\lambda \tau)}\right)\right|_{t \rightarrow t+\lambda \tau} \\
& \times g_{\beta}^{(\nu)}(x, t+\lambda \tau) h(x, t) d x d t .
\end{aligned}
$$

Taking into account that

$$
\int_{t_{0}-\tau}^{t_{0}} \int_{\Omega} h(x, t) d x d t=\int_{t_{1}}^{t_{1}+\tau} \int_{\Omega} h(x, t) d x d t \quad \forall h \in D\left(N_{u}^{\prime}\right) .
$$

the equality (22) can be written as

$$
\begin{aligned}
(23)\left\langle N_{u}^{\prime} h, g\right\rangle= & \int_{t_{0}}^{t_{1}} \int_{\Omega} \sum_{\lambda=-1}^{1} \sum_{k, \nu=0}^{l} \sum_{|\alpha|,|\beta|=0}^{s}(-1)^{|\alpha|+k} C_{k}^{\nu}\left(\begin{array}{c}
\alpha \\
\beta
\end{array}\right) \\
& \times D_{t}^{k-\nu} D_{\alpha-\beta}\left(\frac{\partial f}{\partial u_{\alpha}^{(k)}(x, t+\lambda \tau)} g(x, t+\lambda \tau)\right) h(x, t) d x d t .
\end{aligned}
$$


Bearing in mind the equality (19) we obtain

$$
\begin{aligned}
\left\langle N_{u}^{\prime} h, g\right\rangle= & \int_{t_{0}}^{t_{1}} \int_{\Omega} \sum_{\lambda=-1}^{1} \sum_{\nu=0}^{l} \sum_{|\beta|=0}^{s}\left(\frac{\partial f}{\partial u_{\beta}^{(\nu)}(x, t-\lambda \tau)}\right) \\
& \times\left(g_{\beta}^{(\nu)}(x, t-\lambda \tau)\right) h(x, t) d x d t .
\end{aligned}
$$

By using (23), (24) we get

$$
\begin{aligned}
&\left\langle N_{u}^{\prime} h, g\right\rangle-\left\langle N_{u}^{\prime} g, h\right\rangle= \int_{t_{0}}^{t_{1}} \int_{\Omega} \sum_{\lambda=-1}^{1} \sum_{k, \nu=0}^{l_{0}} \sum_{|\alpha|,|\beta|=0}^{s}\left[(-1)^{|\alpha|+k+\nu} C_{k}^{\nu}\left(\begin{array}{l}
\alpha \\
\beta
\end{array}\right)\right. \\
& \times\left. D_{t}^{k-\nu} D_{\alpha-\beta}\left(\frac{\partial f}{\partial u_{\alpha}^{(k)}(x, t-\lambda \tau)}\right)\right|_{t \rightarrow t+\lambda \tau} \\
&\left.-\frac{\partial f}{\partial u_{\beta}^{(\nu)}(x, t-\lambda \tau)}\right] g_{\beta}^{(\nu)}(x, t+\lambda \tau) h(x, t) d x d t \\
& \forall u \in D(N), \forall g, h \in D\left(N_{u}^{\prime}\right) .
\end{aligned}
$$

Since $g$ and $h$ are arbitrary functions from $D\left(N_{u}^{\prime}\right)$ then $\left\langle N_{u}^{\prime} h, g\right\rangle-$ $\left\langle N_{u}^{\prime} g, h\right\rangle=0 \forall u \in D(N), \forall h, g \in D\left(N_{u}^{\prime}\right)$ if and only if conditions (18) hold.

Example 2. Let us consider the equation

$$
\begin{aligned}
&(25) N_{1}(u) \equiv \sum_{\lambda=-1}^{1}\left(a_{\lambda} u_{t t}(x, t+\lambda \tau)-b_{\lambda}^{i j} u_{x_{i} x_{j}}(x, t+\lambda \tau)\right)=0, \\
&(x, t) \in Q=\Omega \times\left(t_{0}, t_{1}\right), t_{1}-t_{0}>2 \tau .
\end{aligned}
$$

Here $u=u(x, t)$ is an unknown function, $u_{t t}=\frac{\partial^{2} u}{\partial t^{2}}, u_{x_{i}, x_{j}}=\frac{\partial^{2} u}{\partial x_{i} \partial x_{j}} ; \quad a_{\lambda} \in$ $C_{x, t}^{0,2}(\bar{Q}), b_{\lambda}^{i j} \in C_{x, t}^{2,0}(\bar{Q}), \Omega$ is a bounded domain in $R^{m}$ with a piecewise smooth boundary $\partial \Omega ; \bar{\Omega}$ is the closure of $\Omega$ in $R^{m}$, and repeated indices of factors situated at different levels denote summation, $i, j=\overline{1, m}$.

Let us define the domain of definition of $N_{1}$ by setting

$$
\begin{aligned}
D\left(N_{1}\right)= & \left\{u \in U=C^{2}\left(\overline{Q_{\tau}}\right):\right. \\
& \frac{\partial^{k} u(x, t)}{\partial t^{k}}=\varphi_{1 k}(x, t),(x, t) \in E_{1}=\bar{\Omega} \times\left[t_{0}-\tau, t_{0}\right], k=0,1,
\end{aligned}
$$




$$
\begin{aligned}
& \frac{\partial^{k} u(x, t)}{\partial t^{k}}=\varphi_{2 k}(x, t),(x, t) \in E_{2}=\bar{\Omega} \times\left[t_{1}, t_{1}+\tau\right], k=0,1, \\
& \left.\left.\frac{\partial u}{\partial n_{x}}\right|_{\Gamma_{\tau}}=\psi(x, t)\right\},
\end{aligned}
$$

where $\Gamma_{\tau}=\partial \Omega \times\left[t_{0}-\tau, t_{1}+\tau\right], Q_{\tau}=\Omega \times\left(t_{0}-\tau, t_{1}+\tau\right), \varphi_{i 0} \in C^{1}\left(E_{i}\right), \varphi_{i 1}=$ $\frac{\partial \varphi_{i 0}}{\partial t}(i=1,2), \psi \in C\left(\Gamma_{\tau}\right)$.

First, let us study the existence of the classical variational formulation of problem (25), (26). For that aim, we introduce the notation $V=C\left(\overline{Q_{\tau}}\right)$ and use the bilinear form (17).

The conditions of potentiality (18) for the given case take the form

$$
\begin{aligned}
a_{-\lambda}(x) & =a_{\lambda}(x), \quad x \in \Omega, \quad \lambda=-1,0,1, \\
\left.b_{-\lambda}^{i j}(t)\right|_{t \rightarrow t+\lambda \tau} & =b_{\lambda}^{i j}(t), \quad t \in\left[t_{0}, t_{1}\right], \quad \lambda=-1,0,1, \quad i, j=\overline{1, m} .
\end{aligned}
$$

If this relations are hold the given problem (25), (26) allows the classical variational formulation and the corresponding functional has the form

$$
\begin{aligned}
F_{N}[u]= & -\frac{1}{2} \int_{t_{1}}^{t_{2}} \int_{\Omega}\left[a_{1}(x) u_{t}(x, t-\tau) u_{t}(x, t)+a_{0}(x) u_{t}^{2}(x, t)\right. \\
& +a_{1}(x) u_{t}(x, t+\tau) u_{t}(x, t)+b_{1}^{i j}(t-\tau) u_{x_{i}}(x, t-\tau) u_{x_{j}}(x, t) \\
& \left.+b_{0}^{i j}(t) u_{x_{i}}(x, t) u_{x_{j}}(x, t)+b_{1}^{i j}(t+\tau) u_{x_{i}}(x, t+\tau) u_{x_{j}}(x, t)\right] d x d t \\
& + \text { const. }
\end{aligned}
$$

Example 3. Let us consider the equation

(27) $N_{2}(u) \equiv b\left(u_{x}\right)^{2}(x, t+\tau)-a\left(u_{t}\right)^{2}(x, t)-2 a u_{t t}(x, t)$

$$
\begin{gathered}
-2 b e^{u(x, t-\tau)-u(x, t)}\left\{u_{x}(x, t-\tau) u_{x}(x, t)+u_{x x}(x, t)\right\}=0, \\
(x, t) \in Q=(c, d) \times\left(t_{1}, t_{2}\right), t_{2}-t_{1}>2 \tau, \tau>0,
\end{gathered}
$$

where $a, b$ are constants, $u$ is an unknown function, $u_{x}=\frac{\partial u}{\partial x}, u_{t}=\frac{\partial u}{\partial t}, u_{t t}=$ $\frac{\partial^{2} u}{\partial t^{2}}$.

Let us definite the domain of definition of $N_{2}$ by setting

$$
\begin{aligned}
D\left(N_{2}\right)=\{ & \left\{u \in=C_{x, t}^{2,2}\left(\overline{Q_{\tau}}\right):\right. \\
& \frac{\partial^{k} u(x, t)}{\partial t^{k}}=\varphi_{1 k}(x, t),(x, t) \in E_{1}=[c, d] \times\left[t_{1}-\tau, t_{1}\right] ; k=0,1,
\end{aligned}
$$




$$
\begin{aligned}
& \frac{\partial^{k} u}{\partial t^{k}}=\varphi_{2 k}(x, t), \quad(x, t) \in E_{2}=[c, d] \times\left[t_{2}, t_{2}+\tau\right] ; k=0,1, \\
& \left.u(c, t)=\psi_{1}(t), \quad u(d, t)=\psi_{2}(t), t \in\left[t_{1}-\tau, t_{2}+\tau\right]\right\}
\end{aligned}
$$

where $Q_{\tau}=(c, d) \times\left(t_{1}-\tau, t_{2}+\tau\right), \varphi_{i 0} \in C^{1}\left(E_{i}\right), \varphi_{i 1}=\frac{\partial \varphi_{i 0}}{\partial t}, \psi_{i} \in$ $C\left(\left[t_{1}-\tau, t_{2}+\tau\right]\right)(i=1,2)$.

It is easy to check that operator (27) is not potential on the domain (28) with respect to the classical bilinear form (17). In that connection we search for the function $M=M\left(x, t, u, u_{t}\right) \neq 0$ and the functional $F_{N}[u]$ such that

$\delta F[u, h]=\int_{t_{1}}^{t_{2}} \int_{\Omega} M\left(x, t, u, u_{t}\right) N(u) \delta h d x d t \quad \forall u \in D(N), \quad \forall h \in D\left(N_{u}^{\prime}\right)$.

Using the theorem 2 it is easy to find that $M=e^{u(x, t)}$. The operator $N(u)=e^{u(x, t)} N_{2}(u)$ is potential.

The corresponding functional is given by

$$
F_{N}[u]=\int_{t_{1}}^{t_{2}} \int_{\Omega} e^{u(x, t-\tau)}\left[a\left(u_{t}\right)^{2}(x, t-\tau)+b\left(u_{x}\right)^{2}(x, t)\right] d x d t
$$

Remark 1. Differential equations with several variable deviating arguments occur for example in the technical cybernetics (see [11]).

Remark 2. The theorem 1 generalizes appropriate results by Popov [7].

Remark 3. Different approaches to the definition of solutions of different types of functional differential equations are discussed by Hale [3].

Remark 4. The study of the inverse problems of the calculus of variations for equations with deviating arguments has been initiated by Savchin [10].

Acknowledgements. This research was partially supported by the Russian Fund of Basic Research under grant no.05.01.00422A.

\section{References}

[1] L. E. El'sgol'tz, Qualitative methods in mathematical analysis, Trans. Math. Mono., Amer. Math. Soc., 12 (1964).

[2] V. M. Filippov, V. M. Savchin and S. G. Shorokhov, Variational principles for nonpotential operators, J. Math. Sci, 68 (3) (1994), 275398. 
[3] J. Hale, Theory of Functional Differential Equations, Springer-Verlag, New York, 1977.

[4] H. Helmholtz, Ueber die physikalische bedeutung des prinzips der kleinsten wirkung, J.Reine und Angew. Math. 100 (1887), 137-166.

[5] D. K. Hughes, Variational and optimal control problem with delayed arguments, J. Optimization Theory Appl., 2 (1968), 1-14.

[6] G. A. Kamenskii, Variational and boundary-value problems with deviating argument, Differents. Uravn, 69 (8) (1970), 1349-1358.

[7] A. M. Popov, Conditions for potentiality of differential difference equations, Differents. Uravn., 34 (3) (1998), 422-424.

[8] L. D. Sabbagh, Variational problems with lags, J. Optimization Theory Appl., 3 (1969), 34-51.

[9] R. M. Santilli, Foundations of Theoretical Mechanics 1, The Inverse Problem in Newtonian Mechanics, Springer-Verlag, 1978.

[10] V. M. Savchin, Helmholtz's conditions of potentiality for PDE with deviating arguments, in Theses of the 35th scientific conference of the departement of physico-mathematical and natural science, 1994, May 16-24, Part 2, Mathematical sections. - M.:RUDN, 1994, p. 25.

[11] A. V. Solodov and E. A. Solodova, Systems with variable retardation, [in Russian] Nauka, Moscow, 1980.

[12] E. Tonti, A general solution of the inverse problem of the calculus of variations, Hadronic J., 5 (4) (1982), 1404-1450.

[13] V. Volterra, Lecons sur les Fonctions de Lignes, Paris, Gautier-Villars, 1913.

Peoples' Friendship University of Russia

Miklukho-Maklaya str., 6

117198, Moscow

Russia

(E-mail:VSavchin@yandex.ru

Email: IKolesnikova@sci.pfu.edu.ru

E-mail: AMPopov@sci.pfu.edu.ru)

(Received: August 2005) 


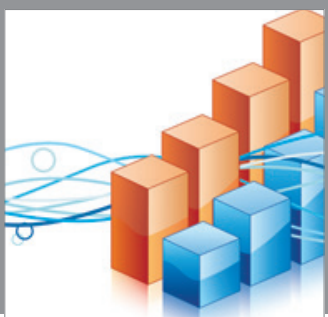

Advances in

Operations Research

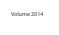

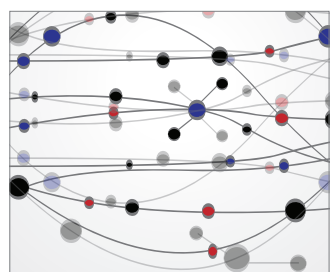

\section{The Scientific} World Journal
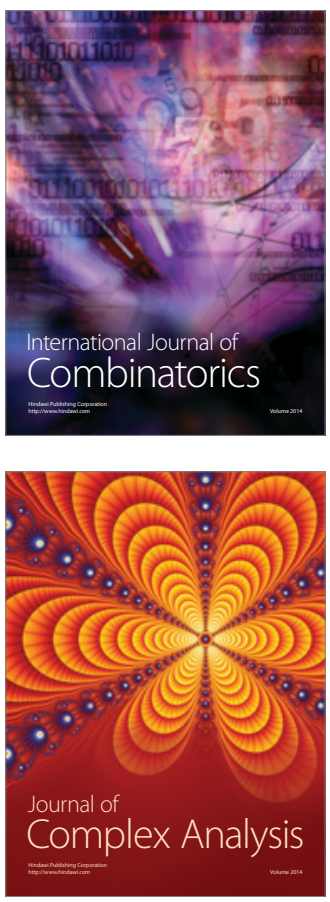

International Journal of

Mathematics and

Mathematical

Sciences
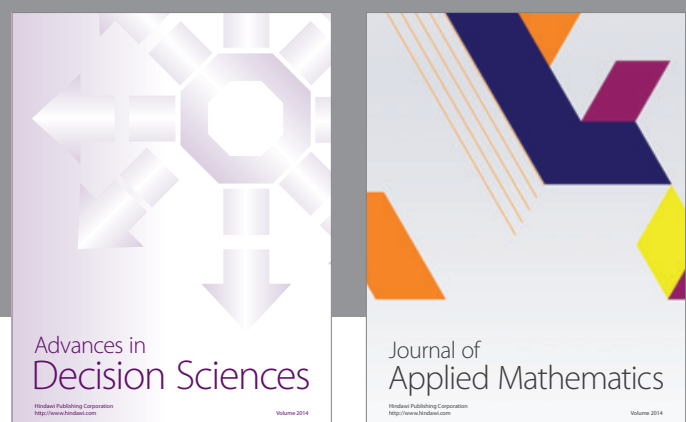

Journal of

Applied Mathematics
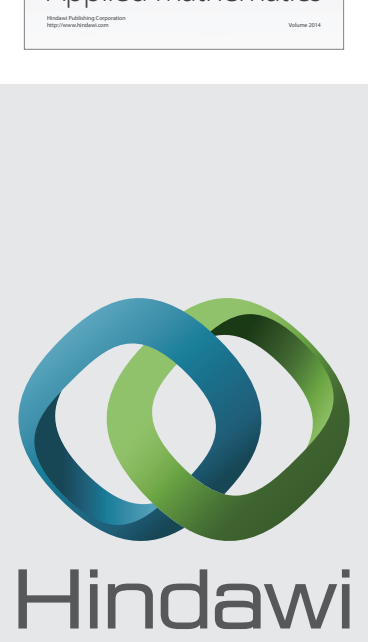

Submit your manuscripts at http://www.hindawi.com
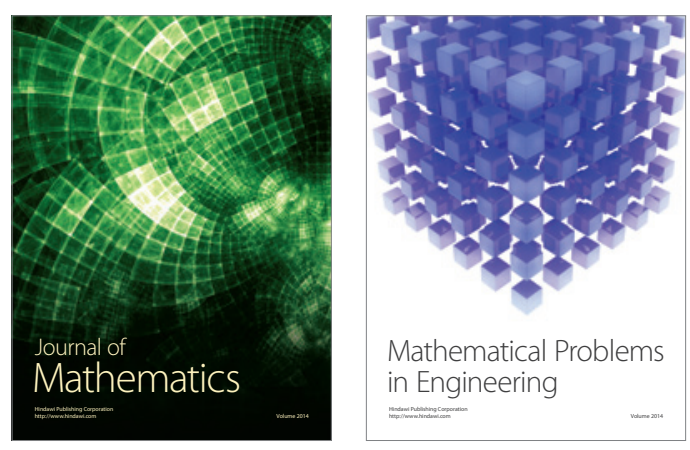

Mathematical Problems in Engineering
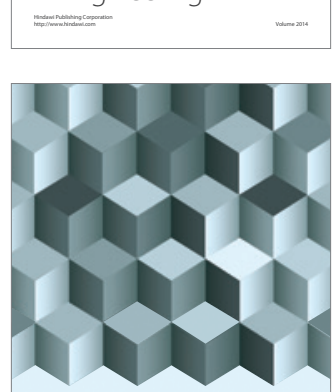

Journal of

Function Spaces
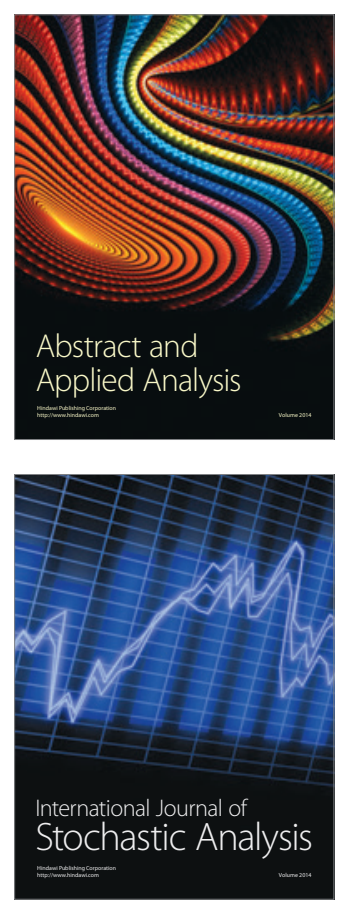

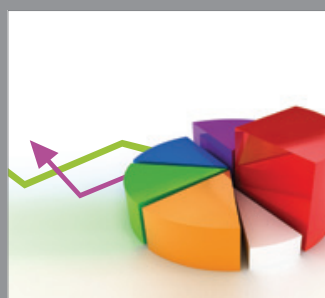

ournal of

Probability and Statistics

Promensencen
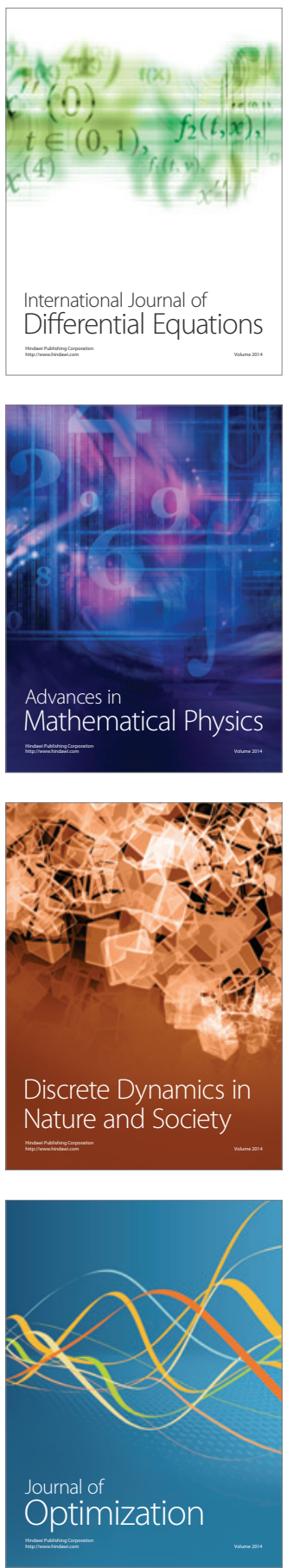